2014-03

Familial strife on the seashore:

\title{
Aggression increases with relatedness in the sea anemone Actinia equina
}

Foster, Nicola

http://hdl.handle.net/10026.1/3690

10.1016/j.beproc.2014.01.009

Behavioural Processes

Elsevier BV

All content in PEARL is protected by copyright law. Author manuscripts are made available in accordance with publisher policies. Please cite only the published version using the details provided on the item record or document. In the absence of an open licence (e.g. Creative Commons), permissions for further reuse of content should be sought from the publisher or author. 
Familial strife on the seashore: Aggression increases with relatedness in the sea anemone Actinia equina

Nicola L. Foster* and Mark Briffa

Marine Biology and Ecology Research Centre, Plymouth University, Drake Circus, Plymouth, PL4 8AA, UK

Email addresses: mark.briffa@ plymouth.ac.uk, nicola.foster@plymouth.ac.uk *corresponding author: Tel.: + 44 (0)1752 584598 


\begin{abstract}
Pairwise contests occur when two individuals compete directly over ownership of an indivisible resource. Contests vary in the degree of escalation, some encounters being settled through non-injurious behaviour while others are only resolved after dangerous fighting. Here, we investigate the role of relatedness, assessed using AFLP analysis, on the occurrence of stinging during staged contests in the beadlet sea anemone Actinia equina. Contrary to our expectations, we found that the chance of stinging, and hence the chance of inflicting damage, increased with the degree of relatedness between the two opponents. This result may be explained by the negative relationship between asymmetry in fighting ability and escalation level predicted by theory. We suggest that in order to fully understand how relatedness influences aggression, predictions from kin selection theory should be incorporated with those from contest theory.
\end{abstract}

Keywords Actinia equina; AFLP; aggression; contest; relatedness; 


\section{Introduction}

Contest behaviour occurs when individuals directly interact during competition over the ownership of an indivisible resource unit. Contests are often settled through the use of noninjurious agonistic behaviour. In other cases, where the value of the resource is especially high or when the opponents are evenly matched, the level of aggression may escalate and a dangerous fight becomes more likely (Briffa \& Hardy 2013). While the roles of resource value and fighting ability ('resource holding potential', RHP) have received much attention (Briffa \& Hardy 2013), we should also expect escalation patterns to vary with the degree of relatedness between the opponents. Kin selection theory implies either a unidirectional negative relationship between relatedness and aggression (Hamilton 1964) or a dome shaped relationship if relatives compete on a very localised scale within isolated groups (Gardner \& West 2004). While these predictions have been supported by empirical studies (e.g. Holmes 1986, Walls \& Roudebush 1991, Ensminger \& Meikle 2005, Sato et al. 2013), there are also examples where these effects are absent (e.g. Tóth et al. 2009, Hirsch et al. 2012). Where kinship does not appear to moderate aggression, presumably the direct fitness benefits of acquiring a resource appear to outbalance the indirect benefits of avoiding damage to a relative. Alternatively, kin recognition may be weak or absent.

Thus far, studies investigating the links between kinship and aggression have focussed on examples of contests over reproductive rights in group living species. Contests, however, occur across a very broad range of contexts. Sea anemones are generally asocial (but see Ayer \& Grosberg 2005 for a notable exception) yet highly abundant on rocky shores. In the beadlet anemone, Actinia equina, both sexes engage in contests over space on the substrate. Reproduction is frequently asexual and there are no known differences in fighting behaviour between the sexes (Manuel 1988). Contests can be settled either following low intensity contact or after an escalated fight. In the latter case, one or both opponents use specialised tentacles, acrorhagi, to inflict damaging 'stings' on their rival. Contact with the opponent triggers the discharge of specialised structures called nematocytes embedded in the acrorhagial epithelium. The harpoon-like projections of the nematocytes pierce the recipient's epithelium, delivering a toxic payload. This also results in the tearing of the aggressor's acrorhagi, and distinctive 'peels' are thus left on the opponent. Although, anemones have a simple nervous system, similar to other fighting animals the contest ends when one individual makes a decision to retreat. Thus, sea anemones represent an ideal study system in which to investigate the link between relatedness and aggression in animals. While recent studies have 
revealed much about the decision to withdraw (Rudin \& Briffa 2011), little is known about the decision to escalate a contest in anemones. A. equina are known to behave passively towards clonemates, and aggressively towards non-clonemates (Turner et al. 2003). However, it is not clear whether they recognise all non-clonemates as non-self (Turner et al. 2003). If anemones do show kin recognition, kin selection theory indicates that individuals should be less aggressive towards closer relatives. We would therefore expect to see a unidirectional negative relationship with the degree of relatedness between rivals during pairwise contests.

\section{Material and Methods}

A. equina were collected inter-tidally from The Hoe (Plymouth, UK) during November 2011 and January 2012 and transported to the laboratory within $2 \mathrm{~h}$ of collection. Only anemones of the red/brown colour morph from the mid/upper shore were used in this study because they have been shown to be more aggressive than green/orange morphs and individuals found on the lower shore (Manuel 1988). As in previous studies, we assumed that individuals collected $>1 \mathrm{~m}$ apart on the shore would not be clone mates. In the laboratory, anemones were placed individually on flat stones, size matched by visual estimation and housed in pairs of a similar size in plastic tanks containing $700 \mathrm{ml}$ of aerated seawater at $15 \pm 0.5{ }^{\circ} \mathrm{C}$. Paired anemones were separated by plastic netting used to divide the tank in half. The seawater was changed every 2-3 days and the anemones were fed aquaria marine fish flakes every 2-3 days.

Paired anemones ( $n=15$ pairs) were transferred to opposite ends of a new tank (as above) containing fresh seawater and allowed $5 \mathrm{~min}$ to settle. The 2 anemones were then moved to the centre of the tank such that they were in tentacle contact with each other. This was defined as the starting point of the contests. The contests were recorded using a Sony easycam camera. At the end of the encounter, contests were categorised according to level of escalation; $0=$ no stings, $1=$ one anemone stings, $2=$ both anemones sting. Following conclusion of each contest, a small piece of tissue (approximately $1 \mathrm{~cm} \times 0.5 \mathrm{~cm}$ ) was removed from the pedal disc of each anemone and stored in $100 \%$ ethanol at $4{ }^{\circ} \mathrm{C}$ prior to analysis. To quantify the degree of relatedness between each pair of anemones we used amplified fragment length polymorphism (AFLP) analysis across 56 individuals. See Supplement 1 for details.

In order to estimate relatedness between the paired individuals that engaged in contests, pairwise similarity distances were calculated using the Jaccard coefficient in Primer 6 v6.1.13 
(Clarke and Gorley 2006). A binary logistic regression was used to determine the effect of relatedness on the chance of escalation ( 1 or 0 ). This test was carried out in $\mathrm{R}$ using the lme4 package.

\section{Results}

Relatedness ranged from 11.6 to 76.9 and the mean similarity distance was $42.2 \pm$ S.E $=4.7$. Of the 15 staged contests, 11 were resolved without either individual stinging its opponent (escalation level $=0$ ) and in the remaining 4 contests, stings were inflicted by one opponent (escalation level $=1$ ). The likelihood of stinging increased with relatedness between the opponents $\left(\chi_{1}^{2}=4.9, \mathrm{P}=0.027\right.$; Figure 1$)$.

\section{Discussion}

Kin selection theory indicates that animals might show reduced levels of aggression towards close relatives, since any damage inflicted on a relative could reduce the indirect component of one's own fitness. On the other hand, this effect would be absent if kin-recognition is limited or if the direct fitness benefits of access to a limiting resource outbalance the indirect fitness that accrues from reduced aggression towards relatives. Thus, there may be no effect of relatedness on aggression, as in house sparrows (Tóth et al. 2009) and ring tailed coatis (Hirsch et al. 2012). Here, rather than the absence of a relatedness effect, we found the surprising result of a positive relationship between relatedness and aggression, with fighting anemones showing an extreme disregard for the wellbeing of relatives.

This seemingly paradoxical result could be explained by the ecology of $A$. equina and by contest theory. First, as noted above, A. equina may be good at recognising self from nonself but poor at recognising kin (Turner et al. 2003). Nevertheless, escalated contests are frequently observed in the field, indicating the importance of aggression for resource acquisition. Maynard Smith \& Price (1973) demonstrated how natural selection should produce a mixture of dangerous and non-dangerous agonistic behaviour, with the proportion of non-dangerous behaviour increasing as the cost of an injury increases relative to the value of the resource. Thus, we might expect closer relatives to be more aggressive to one another, if relatedness co-varies with the subjective value that they place on the resource. Resource value, however, is not the only factor expected to drive the level of escalation. Contests are resolved quickly, without recourse to dangerous fighting, when the disparity in RHP between 
opponents is high. For a given resource value, it is only when opponents are closely matched, that highly escalated contests are likely. Therefore, we might also expect closer relatives to be more aggressive, if relatedness co-varies with the expression of RHP traits. In A. equina, RHP increases with body size, nematocyst length (Rudin \& Briffa 2011) and boldness (Rudin $\&$ Briffa 2012). In this study opponents were size matched and further studies examining the interaction between these RHP traits, variation in the motivation to fight, and relatedness are therefore warranted. Although this result derives from a relatively low number of staged encounters, we provide the first evidence of the potential for a positive relationship between relatedness and aggression, within a population of asocial animals competing over space. This result may be due to co-variation between relatedness and a range of traits known to influence escalation in contests.

\section{Acknowledgements}

We are grateful to Ann Tor, Marie Hawkins and Lizzie Edmonds for assistance with the lab set-up and with collecting anemones.

\section{References}

Ayer, D.J \& Grosberg, R.K. 2005. Behind anemone lines: factors affecting division of labour in the social cnidarian Anthopleura elegantissima. Anim Behav 70: 97-110.

Briffa, M. and Hardy, I.C.W. 2013. Introduction to Animal Contests. In Hardy. I.C.W. \& Briffa, M. (Eds). Animal Contests. Cambridge University Press.

Clarke, K.R. and Gorley, R.N. 2006. PRIMER v6: User Manual/Tutorial. PRIMER-E, Plymouth, 192 pp.

Ensminger, A.L. \& Meikle, D.B. 2005. Effects of male kinship and agonistic behaviour on reproduction and odour preferences of female house mice, Mus domesticus. Anim Behav 69:1147-1155.

Gardner, A., \&S. A. West. 2004. Spite and the scale of competition. J Evol Biol 17:11951203. 
Hamilton, W.D. 1964 The genetical evolution of social behaviour. I, II. J Theor Biol 7:1-52.

Hirsch, B.T., Stanton, M.A., \& Maldonado, J.E. 2012. Kinship shapes affiliative social networks but not aggression in ring-tailed coatis. PLoS One 7: e37301.

Holmes, W.G. 1986. Kin recognition by phenotype matching in female Belding's ground squirrels. Anim Behav 34:38-47.

Manuel, R.L. 1988. British Anthozoa. Academic Press, London.

Maynard Smith, J. \& Price, G.R. 1973. The logic of animal conflict. Nature, 246: 15-18.

Rudin, F.S. \& Briffa, M. 2011. The logical polyp: assessments and decisions during contests in the beadlet anemone Actinia equina. Behavioral Ecology, 22: 1278-1285.

Rudin, F.S. \& Briffa M. 2012. Is boldness a Resource Holding Potential trait? Fighting prowess and changes in startle response in the sea anemone Actinia equina. Proc $R$ Soc Lond B 279: 1904-1910.

Sato, Y., Egas, M., Sabelis1, M.W. \& Mochizuki, A. 2013. Male-male aggression peaks at intermediate relatedness in a social spider mite. Ecol Evol 3: 2661- 2669.

Tóth, Z., Bókony, V., Lendvai, Á.Z., Szabó, K. \& Pénzes, Z. \& Liker, A. 2012. Kinship and aggression: do house sparrows spare their relatives? Behav Ecol Sociobiol 63:1189-1196.

Turner, V.L.G., Lynch S.M., Paterson L., León-Cortés, J.L. \& Thorpe J. P. 2003. Aggression as a function of genetic relatedness in the sea anemone Actinia equina (Anthozoa: Actiniaria). MEPS 247: 85-92.

Walls, S.C. \& Roudebush, R.E. 1991. Reduced aggression toward siblings as evidence of kin recognition in cannibalistic salamanders. Am Nat 138:1027-1038. 


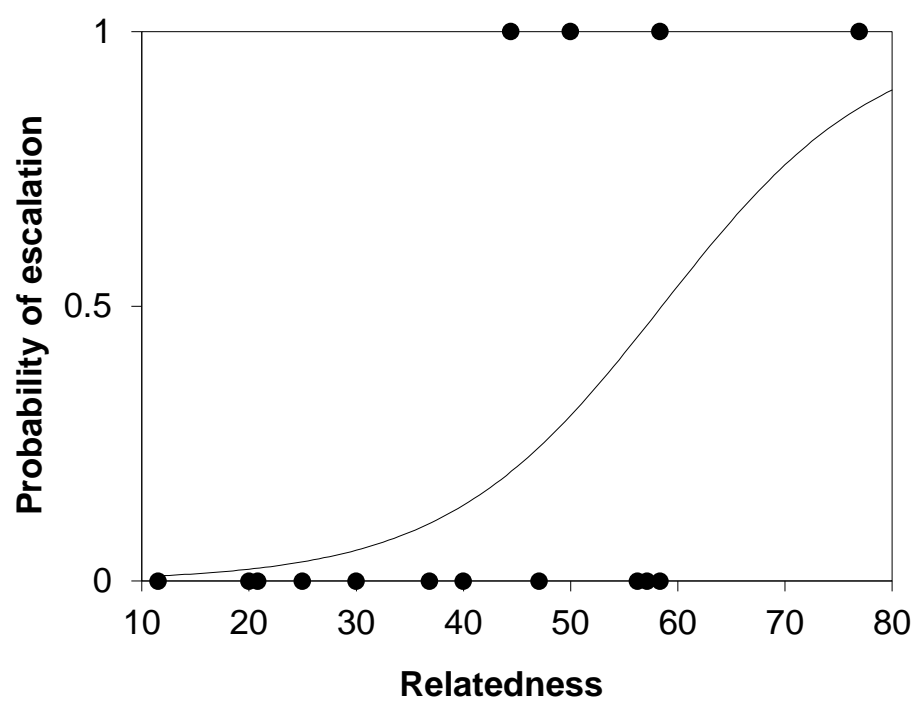

Figure 1: The effect of relatedness (Jaccard coefficient) on the probability of an escalated fight involving stinging in the sea anemone Actinia equina. Individual data points are represented by solid circles.

\section{Supplement1: Details of AFLP methods}

\section{DNA extraction and sequencing}

DNA was extracted from the pedal disc tissue of the 30 anemones used in contests plus an additional 26 individuals from the same shore, in order to increase the power of the AFLP analysis. DNA extraction and AFLP analysis DNA was extracted from anemone tissue ( $\mathrm{n}=$ 56) using a DNeasy Kit (Qiagen, UK) following the manufacturer's instructions. DNA was quantified using a Nanodrop ND 1000 spectrophotometer and samples were standardised to $100 \mathrm{ng} \mu \mathrm{L}^{-1}$. To assess reproducibility of AFLP fragments (Bonin et al. 2004), 5\% of samples were run repeatedly in different PCR reactions. Four sets of primers were used for the AFLP analysis (Table S1) and EcoRI and MseI adapters (Table S1) were prepared by heating a 5 $\mu \mathrm{M}$ stock of each adaptor for $5 \mathrm{~min}$ at $95{ }^{\circ} \mathrm{C}$ and then cooling to room temperature.

Restriction-ligation was conducted by combining $200 \mathrm{ng}$ of template DNA, $2 \mathrm{mM}$ ATP, $100 \mathrm{ng} \mathrm{LL}^{-1} \mathrm{BSA}, 0.05 \mathrm{M} \mathrm{NaCl}, 10 \mathrm{U}$ each of EcoRI and MseI enzymes, $100 \mathrm{U}$ of T4 DNA ligase, $5 \mathrm{pM}$ of each double stranded adaptor, $1 \mathrm{X}$ T4 DNA ligase buffer, and made up to a total volume of $30 \mu \mathrm{L}$ using molecular grade $\mathrm{H}_{2} \mathrm{O}$. The mixture was incubated at $37{ }^{\circ} \mathrm{C}$ for 3 hours, then $65{ }^{\circ} \mathrm{C}$ for $10 \mathrm{~min}$ to inactivate the enzymes. Pre-selective amplifications contained 1X PCR buffer, $0.2 \mathrm{mM}$ dNTP's, $5 \mu \mathrm{M}$ of each pre-selective primer (EcoRI and MseI; Table S1), 1 U Taq DNA polymerase (Qiagen Taq PCR Core Kit), and $4 \mu \mathrm{L}$ of the 
diluted (1:20) restricted-ligated DNA, in a total volume of $20 \mu \mathrm{L}$. Cycling conditions were as described by Kazachkova, Fahleson, and Meijer (2004). Selective amplification was conducted using the same reagents and concentrations as in the pre-selective step but with 5 $\mu \mathrm{M}$ of each selective primer (each selective primer pair was used in a separate reaction) and $2.5 \mu \mathrm{L}$ of diluted (1:20) pre-selective product in a volume of $10 \mu \mathrm{L}$. Cycling conditions consisted of an initial denaturation step at $94{ }^{\circ} \mathrm{C}$ for $2 \mathrm{~min}$, followed by 13 cycles of $94{ }^{\circ} \mathrm{C}$ for $30 \mathrm{~s}, 65^{\circ} \mathrm{C}$ for $30 \mathrm{~s}$ and $72{ }^{\circ} \mathrm{C}$ for 1 min with a $0.7^{\circ} \mathrm{C}$ reduction in annealing temperature per cycle $\left(65{ }^{\circ} \mathrm{C}\right.$ for the first cycle), and then 23 cycles of $94{ }^{\circ} \mathrm{C}$ for $30 \mathrm{~s}, 56{ }^{\circ} \mathrm{C}$ for $30 \mathrm{~s}$ and $72{ }^{\circ} \mathrm{C}$ for $1 \mathrm{~min}$, and a final elongation step of $72{ }^{\circ} \mathrm{C}$ for $5 \mathrm{~min}$. The AFLP fragments were run separately for each primer pair in a mix of $1 \mu \mathrm{l}$ diluted (1:100) selective amplification product, $0.3 \mu \mathrm{l}$ of LIZ-500 size standard (Applied Biosystems, Paisley, UK) and $15 \mu \mathrm{l}$ of HiDi Formamide on an ABI 3130 sequencer (Applied Biosystems). Samples were denatured at $95^{\circ} \mathrm{C}$ for 2 min before being analysed. All dilutions were made using molecular grade $\mathrm{H}_{2} \mathrm{O}$.

\section{Fragment analysis}

Following Ley and Hardy (2013), AFLP fragments were collated using the Peak Scanner Software v2.0 (Applied Biosystems) and analysed with the size standard and the sizing default PP, which assumes primers have not been removed from the samples. A commaseparated (CSV) table containing the analysed data for each sample was exported, and then used as the input dataset for tinyFLP (Arthofer 2010), an open-source software for automatic scoring and conversion of peak data into a binary allelic matrix. The parameters chosen for scoring differed for each primer set (Table S2). Primer set S3 failed to amplify fragments consistently so was not used in further analysis. TinyCAT (Arthofer 2010) was used to concatenate the binary matrices from the 3 remaining primer sets. A total of 96 polymorphic loci were scored for the 3 primer sets (36, 28 and 32 loci for S1, S2 and S4, respectively) and 56 individuals were genotyped. The duplicated samples revealed a multilocus genotyping error rate of $18.4 \%$.

Table S1: Sequences of AFLP primers, including fluorescent label where appropriate (in bold), used to genotype Actinia equina individuals.

\begin{tabular}{|c|c|c|}
\hline Prime & & Sequence $\left(5^{\prime}-3^{\prime}\right)$ \\
\hline \multirow{4}{*}{ Adapters } & MseI & F - GAC GAT GAG TCC TGA G \\
\hline & & R - TAC TCA GGA CTC AT \\
\hline & & F - CTC GTA GAC TGC GTA CC \\
\hline & EcoRI & R - AAT TGG TAC GCA GTC TAC \\
\hline \multirow{2}{*}{ Pre-selective } & MseI & GAT GAG TCC TGA GTA AC \\
\hline & EcoRI & GAC TGC GTA CCA ATT CC \\
\hline \multirow{2}{*}{ Selective S1 } & MseI & GAT GAG TCC TGA GTA ACA C \\
\hline & EcoRI & 6FAM GAC TGC GTA CCA ATT CAC A \\
\hline \multirow{2}{*}{ Selective S2 } & MseI & GAT GAG TCC TGA GTA ACT T \\
\hline & EcoRI & VIC GAC TGC GTA CCA ATT CAC T \\
\hline \multirow{2}{*}{ Selective S3 } & MseI & GAT GAG TCC TGA GTA ACA A \\
\hline & EcoRI & PET GAC TGC GTA CCA ATT CAC G \\
\hline
\end{tabular}


Selective S4 MseI GAT GAG TCC TGA GTA ACA A

EcoRI NED GAC TGC GTA CCA ATT CAC C

Table S2: Parameters used for scoring peaks within each primer set in tinyFLP (Arthofer 2010).

\begin{tabular}{ccccc} 
Parameter & Primer S1 & Primer S2 & Primer S3 & Primer S4 \\
\hline Minimum peak height & 100 & 100 & 100 & 100 \\
Maximum peak width & 2 & 2 & 2 & 2 \\
Minimum peak size (bp) & 80 & 80 & 80 & 80 \\
Maximum peak size (bp) & 400 & 300 & 350 & 500 \\
Size tolerance & 0.5 & 0.5 & 0.5 & 0.5 \\
Minimum peak-peak difference & 1.5 & 1.5 & 1.5 & 1.5 \\
Peak height difference & 0 & 0 & 0 & 0 \\
Minimum frequency & 0.1 & 0.1 & 0.1 & 0.1 \\
Maximum frequency & 99.9 & 99.9 & 99.9 & 99.9
\end{tabular}

\section{References}

Arthofer, W. 2010. tinyFLP and tinyCAT: software for automatic peak selection and scoring of AFLP data tables. Molecular Ecology Resources, 10: 385-388.

Bonin, A., Bellemain, E., Eidesen, P.B., Pompanon, F., Brochmann, C. and Taberlet, P. 2004. How to track and assess genotyping errors in population genetics studies. Molecular Ecology, 13: 3261-3273.

Kazachkova, N., Fahleson, J. and Meijer, J. 2004. Establishment of the amplified fragment length polymorphism (AFLP) technique for genotyping of pollen beetle (Meligethes aeneus) - a noxious insect pest on oilseed rape (Brassica napus). Molecular Biology Reports, 31: 3742.

Ley, A.C. and Hardy, O.J. 2013. Improving AFLP analysis of large-scale patterns of genetic variation a case study with the Central African lianas Haumania spp (Marantaceae) showing interspecific gene flow. Molecular Ecology, 22: 1984-1997. 